\title{
Uniqueness and error analysis for Hamilton-Jacobi equations with discontinuities
}

\author{
KLAUS DECKELNICK ${ }^{\dagger}$ \\ Institut für Analysis und Numerik, Otto-von-Guericke-Universität Magdeburg, \\ Universitätsplatz 2, 39106 Magdeburg, Germany \\ AND \\ CHARles M. ElliotT ${ }^{\ddagger}$ \\ Department of Mathematics, University of Sussex, Falmer, Brighton BN1 9RF, UK
}

[Received 11 June 2003 and in revised form 10 May 2004]

We consider the Hamilton-Jacobi equation of eikonal type

$$
H(\nabla u)=f(x), \quad x \in \Omega,
$$

where $H$ is convex and $f$ is allowed to be discontinuous. Under a suitable assumption on $f$ we prove a comparison principle for viscosity sub- and supersolutions in the sense of Ishii. Furthermore, we develop an error analysis for a class of finite difference schemes, which are monotone, consistent and satisfy a suitable stability condition.

Keywords: Hamilton-Jacobi equation; viscosity solution; comparison principle; finite difference method; error bounds.

2000 Mathematics Subject Classification: 35F30, 35R05, 65N15.

\section{Introduction}

Let $\Omega \subset \mathbb{R}^{n}$ be a bounded domain with a Lipschitz boundary $\partial \Omega$. We consider the Hamilton-Jacobi equation

$$
\begin{aligned}
H(\nabla u) & =f(x), \quad x \in \Omega, \\
u(x) & =\phi(x), \quad x \in \partial \Omega,
\end{aligned}
$$

where $f$ and $\phi$ are given functions. The equation (1.1) occurs in a variety of applications including geometrical optics, computer vision and etching. In order to motivate the link to propagating fronts, let us suppose for a moment that such a front at time $t$ can be described as the $t$-level set of an auxiliary function $u: \bar{\Omega} \rightarrow \mathbb{R}$, i.e. $\Gamma(t)=\{x \in \Omega \mid u(x)=t\}$. Then, formally, a unit normal $v$ to $\Gamma(t)$ and the corresponding normal velocity $V$ are given by

$$
v=\frac{\nabla u(x)}{|\nabla u(x)|}, \quad V=\frac{1}{|\nabla u(x)|}, \quad x \in \Gamma(t) .
$$

If in addition, $u$ solves (1.1), $f$ is positive and $H$ is homogeneous of degree one, then

$$
V=\frac{1}{|\nabla u(x)|}=\frac{1}{|\nabla u(x)|} \frac{H(\nabla u(x))}{f(x)}=\frac{1}{f(x)} H(v(x)), \quad x \in \Gamma(t),
$$

${ }^{\dagger}$ Email: Klaus.Deckelnick@mathematik.uni-magdeburg.de

‡Email: C.M.Elliott@sussex.ac.uk 
i.e. the front moves with a normal velocity which depends on the properties of the underlying space and the orientation of the front. Furthermore, we can interpret $u(x)$ as the first arrival time at the point $x$ of a front which was the zero level set of $u$ at the beginning of the evolution.

It is often desirable to consider situations in which the function $f$ is allowed to be discontinuous, e.g. in geometrical optics, when light propagates through a layered medium. Thus, we shall be concerned both with the well-posedness of (1.1), (1.2) in the case that $f$ is discontinuous and with the convergence of numerical algorithms which approximate the corresponding solution.

Various ways of defining a solution of $(1.1),(1.2)$ for discontinuous $f$ have been suggested. First, Ishii [8] (see \$2 below) extended the concept of viscosity solution introduced in [5] to the case of discontinuous Hamiltonians. He obtained existence and uniqueness results for an equation of the form $H(x, u(x), \nabla u(x))=0$, in which the Hamiltonian $H(x, u, p)$ is allowed to be discontinuous in $u$. Using this notion of solution, Soravia [15] studies a class of Hamilton-Jacobi equations in a control-theoretic framework and gives necessary and sufficient conditions for uniqueness of solutions of boundary value problems. In [10], Newcomb \& Su introduce a concept of solution for (1.1), 1.2) which is based on the optical length function $L(x, y)$ (see $\$ 2$ below) and which they term Monge solution. For lower semicontinuous $f$ they prove a comparison principle as well as existence and uniqueness for the Dirichlet problem. Recently, a further definition of solution was suggested in [4] by Camilli \& Siconolfi for Hamilton-Jacobi equations of the form $H(x, D u)=0$. They introduce a generalized notion of viscosity solution, which allows measurable dependence of $H$ on $x$. In the case of the eikonal equation, i.e. $H(p)=|p|$, this definition involves the measuretheoretic notion of an approximate limit for a subsolution and an essential limit for a supersolution and hence is not symmetric. Comparison and uniqueness results are provided.

Problem (1.1), (1.2) also occurs in shape-from-shading. In this case, the right hand side $f$ is related to the light intensity which can be discontinuous. In [16], Tourin establishes a comparison result for equations of the form $H(x, D u)=0$, in which $H$ is allowed to be discontinuous along a smooth surface. For the shape-from-shading problem, Rouy \& Tourin [13] present a consistent and monotone scheme along with numerical calculations. A related problem is studied in [12], where the unique solution is obtained as the limit of sequences which arise from a suitable regularization of the intensity function.

Our work is based on Ishii's definition of solution, but let us emphasize that even though we allow discontinuities of $f$ the solutions themselves will be Lipschitz continuous. In order to obtain uniqueness for the solution of (1.1), 1.2 ) an assumption on $f$ is needed: this condition (see (F2) below) can be seen as a generalization of a condition which appears in [16], and it amounts to a one-sided continuity constraint along a fixed direction at each point in $\Omega$. Under this assumption we are able to prove a comparison result in Theorem 2.3 In $\$ 3$ we analyze a class of numerical schemes which approximate the solution of $(1.1),(1.2)$. Denoting by $h$ the grid size we obtain an order $\mathcal{O}(\sqrt{h})$ for finite difference schemes which are monotone, consistent and satisfy a suitable stability condition. In $\S 4$ we present examples of schemes which satisfy the above requirements, while $\$ 5$ contains numerical tests.

\section{Existence and uniqueness}

Let us start by defining a viscosity solution of (1.1), (1.2). As already mentioned above, we use a concept which was introduced by Ishii in $[8]$ and which is based on upper and lower semicontinuous envelopes. For a given function $v: \Omega \rightarrow \mathbb{R}$ let 


$$
\begin{gathered}
v^{*}(x):=\limsup _{r \rightarrow 0}\left\{v(y) \mid y \in B_{r}(x) \cap \Omega\right\}, \\
v_{*}(x):=\liminf _{r \rightarrow 0}\left\{v(y) \mid y \in B_{r}(x) \cap \Omega\right\} .
\end{gathered}
$$

DEFINITION 2.1 A function $u \in C^{0}(\bar{\Omega})$ is called a viscosity subsolution (resp. supersolution) of 1.1) if for each $\zeta \in C^{\infty}(\Omega)$ : if $u-\zeta$ has a local maximum (resp. minimum) at a point $x_{0} \in \Omega$, then

$$
H\left(\nabla \zeta\left(x_{0}\right)\right) \leqslant f^{*}\left(x_{0}\right) \quad\left(\text { resp. } \geqslant f_{*}\left(x_{0}\right)\right) .
$$

A viscosity solution of 1.1, , 1.2 is then a function $u \in C^{0}(\bar{\Omega})$ which is both a viscosity sub- and supersolution and which satisfies $u(x)=\phi(x)$ for all $x \in \partial \Omega$.

Assumptions on the data. We shall assume that $H: \mathbb{R}^{n} \rightarrow \mathbb{R}$ satisfies:

(H1) $H(0)=0$ and $H(p)>0$ for all $p \in \mathbb{R}^{n} \backslash\{0\}$.

(H2) $H$ is convex.

(H3) $H(p) \rightarrow \infty$ as $|p| \rightarrow \infty$.

Concerning the right hand side we make the following assumptions:

(F1) $f: \Omega \rightarrow \mathbb{R}$ is Borel measurable and there exist $0<m \leqslant M<\infty$ such that

$$
m \leqslant f(x) \leqslant M \quad \forall x \in \Omega .
$$

(F2) For every $x \in \Omega$ there exist $\epsilon_{x}>0$ and $n_{x} \in S^{n-1}$ so that for all $y \in \Omega, r>0, d \in S^{n-1}$ with $\left|d-n_{x}\right|<\epsilon_{x}$ and $y+r d \in \Omega$ we have

$$
f(y+r d)-f(y) \leqslant \omega(|y-x|+r),
$$

where $\omega:[0, \infty) \rightarrow[0, \infty)$ is a continuous, nondecreasing function with $\omega(0)=0$.

For later purposes we shall also formulate a stronger version of this assumption, namely

$(\mathrm{F} 2)^{*}$ There exist $\epsilon>0$ and $K \geqslant 0$ such that for all $x \in \Omega$ there is a direction $n=n_{x} \in S^{n-1}$ with

$$
f(y+r d)-f(y) \leqslant K r
$$

for all $y \in \Omega, d \in S^{n-1}, r>0$ with $|y-x|<\epsilon,|d-n|<\epsilon$ and $y+r d \in \Omega$.

Clearly, (F2) holds at all points $x$ at which $f$ is continuous, but it also allows for certain types of discontinuous behavior as shown by the following

EXAMPLE. Suppose that a surface $\Gamma$ splits $\Omega$ into two subdomains $\Omega_{1}$ and $\Omega_{2}$ such that $f_{\mid \Omega_{1}} \in$ $C^{0}\left(\bar{\Omega}_{1}\right), f_{\mid \Omega_{2}} \in C^{0}\left(\bar{\Omega}_{2}\right)$ and

$$
\lim _{y \rightarrow x, y \in \Omega_{1}} f(y)<\lim _{y \rightarrow x, y \in \Omega_{2}} f(y) \quad \text { for all } x \in \Gamma .
$$

In addition, assume that the following uniform cone property holds: for every $x \in \Gamma$ there exists a neighborhood $U_{x}$ and a cone $C_{x}$ (which is congruent to a fixed given cone $C_{0}$ ) such that $y \in U_{x} \cap \bar{\Omega}_{1}$ implies that $y+C_{x} \subset \Omega_{1}$. Then (F2) holds with $n=n_{x}$ given by the direction of the cone $C_{x}$. 
To see this, observe that the cone condition prevents a situation where $y \in \bar{\Omega}_{1}, y+r d \in \Omega_{2}$, which would lead to a violation of (F2) (cf. [16], where $\Gamma$ is assumed to be smooth).

One can also consider e.g. a two-dimensional domain $\Omega$, where three curves of discontinuity meet at a triple junction. A suitable modification of the above also yields an example for $(\mathrm{F} 2)^{*}$.

In order to describe our assumptions on $\phi$ let us define $L: \bar{\Omega} \times \bar{\Omega} \rightarrow \mathbb{R}$ by

$$
L(x, y):=\inf \left\{\int_{0}^{1} N\left(f^{*}(\gamma(t)), \gamma^{\prime}(t)\right) \mathrm{d} t \mid \gamma \in W^{1, \infty}((0,1) ; \bar{\Omega}) \text { with } \gamma(0)=x, \gamma(1)=y\right\}
$$

where

$$
N(r, \zeta):=\sup \{-(\zeta, p) \mid H(p)=r\} .
$$

We then suppose the following compatibility condition for the boundary data,

$$
\phi(x)-\phi(y) \leqslant L(x, y) \quad \text { for all } x, y \in \partial \Omega .
$$

We shall assume (H1)-(H3) throughout the paper, while the corresponding assumptions on $f$ and $\phi$ will be stated explicitly.

THEOREM 2.2 Assume that (F1) and (C) hold. Then there exists a viscosity solution $u \in C^{0,1}(\bar{\Omega})$ of (1.1), 1.2.

Proof. We regularize $f$ using the sup-convolution, i.e.

$$
f_{\epsilon}(x):=\sup _{y \in \Omega}\left\{f(y)-\frac{1}{\epsilon}|x-y|^{2}\right\}, \quad \epsilon>0 .
$$

Clearly, $f_{\epsilon}$ is continuous and satisfies $f^{*}(x) \leqslant f_{\epsilon}(x)$ for all $x \in \Omega$. In view of assumption (C) and the monotonicity of $N$ in the first variable we deduce that

$$
\phi(x)-\phi(y) \leqslant L_{\epsilon}(x, y) \quad \forall x, y \in \partial \Omega,
$$

where

$$
L_{\epsilon}(x, y):=\inf \left\{\int_{0}^{1} N\left(f_{\epsilon}(\gamma(t)), \gamma^{\prime}(t)\right) \mathrm{d} t \mid \gamma \in W^{1, \infty}((0,1) ; \bar{\Omega}) \text { with } \gamma(0)=x, \gamma(1)=y\right\} .
$$

Therefore, the problem

$$
\begin{aligned}
H\left(\nabla u^{\epsilon}\right) & =f_{\epsilon}(x), \quad x \in \Omega, \\
u^{\epsilon}(x) & =\phi(x), \quad x \in \partial \Omega,
\end{aligned}
$$

has a unique viscosity solution $u^{\epsilon}$, which is given by the formula

$$
u^{\epsilon}(x)=\inf _{y \in \partial \Omega}\left\{L_{\epsilon}(x, y)+\phi(y)\right\} .
$$

It is not difficult to verify that

$$
\left\|u^{\epsilon}\right\|_{C^{0,1}(\bar{\Omega})} \leqslant C(M, \Omega) \quad \text { uniformly in } \epsilon>0 .
$$


Thus, there exists a sequence $\left(\epsilon_{k}\right)_{k \in \mathbb{N}}$ with $\epsilon_{k} \searrow 0$ as $k \rightarrow \infty$ and $u \in C^{0,1}(\bar{\Omega})$ such that

$$
u^{\epsilon_{k}} \rightarrow u \text { as } k \rightarrow \infty \quad \text { uniformly in } \bar{\Omega} \text {. }
$$

Clearly, $u=\phi$ on $\partial \Omega$. We claim that $u$ is a viscosity solution in the sense of Definition 2.1. Let $\zeta \in C^{\infty}(\Omega)$ and suppose that $u-\zeta$ has a local maximum at $x_{0} \in \Omega$. In view of 2.4 there exist $x_{k} \in \Omega$ such that $x_{k} \rightarrow x_{0}$ as $k \rightarrow \infty$ and $u^{\epsilon_{k}}-\zeta$ has a local maximum at $x_{k}$. Then

$$
H\left(\nabla \zeta\left(x_{k}\right)\right) \leqslant f_{\epsilon_{k}}\left(x_{k}\right)
$$

and taking into account 2.1 we obtain

$$
f_{\epsilon_{k}}\left(x_{k}\right)=\sup _{\left|x_{k}-y\right| \leqslant \sqrt{M \epsilon_{k}}}\left\{f(y)-\frac{1}{\epsilon_{k}}\left|x_{k}-y\right|^{2}\right\} \leqslant \sup \left\{f(y)|| y-x_{0}|\leqslant| x_{k}-x_{0} \mid+\sqrt{M \epsilon_{k}}\right\},
$$

which implies by passing to the limit in 2.5

$$
H\left(\nabla \zeta\left(x_{0}\right)\right) \leqslant \limsup _{k \rightarrow \infty} f_{\epsilon_{k}}\left(x_{k}\right) \leqslant f^{*}\left(x_{0}\right) .
$$

On the other hand, if $u-\zeta$ has a local minimum at $x_{0}$, there exist $\tilde{x}_{k} \in \Omega$ with $\tilde{x}_{k} \rightarrow x_{0}$ as $k \rightarrow \infty$ such that $u^{\epsilon_{k}}-\zeta$ has a local minimum at $\tilde{x}_{k}$. Thus,

$$
H\left(\nabla \zeta\left(\tilde{x}_{k}\right)\right) \geqslant f_{\epsilon_{k}}\left(\tilde{x}_{k}\right) \geqslant f_{*}\left(\tilde{x}_{k}\right) .
$$

Since $f_{*}$ is lower semicontinuous, we deduce that

$$
H\left(\nabla \zeta\left(x_{0}\right)\right) \geqslant f_{*}\left(x_{0}\right) .
$$

In conclusion, $u$ is a viscosity solution of (1.1), 1.2).

Uniqueness of the viscosity solution is a consequence of the following comparison result.

THEOREM 2.3 Assume that (F1), (F2) hold, that $u \in C^{0}(\bar{\Omega})$ is a subsolution of $\overline{1.1}, v \in C^{0}(\bar{\Omega})$ is a supersolution of 1.1 and that at least one of the functions belongs to $C^{0,1}(\bar{\Omega})$. If $u \leqslant v$ on $\partial \Omega$ then $u \leqslant v$ in $\bar{\Omega}$.

Proof. Let us assume that $v \in C^{0,1}(\bar{\Omega})$. We shall use the approach presented in [9] (see also [16]). Fix $\theta \in(0,1)$ and define $u_{\theta}(x):=\theta u(x)$. Next, choose $x_{0} \in \bar{\Omega}$ such that

$$
u_{\theta}\left(x_{0}\right)-v\left(x_{0}\right)=\max _{x \in \bar{\Omega}}\left(u_{\theta}(x)-v(x)\right)=: \mu,
$$

and suppose that $\mu>0$. Upon replacing $u, v$ by $u+k, v+k$, we may assume that $u \geqslant 0$ in $\bar{\Omega}$, so that $u_{\theta} \leqslant u$ in $\bar{\Omega}$. In particular, $u_{\theta} \leqslant v$ on $\partial \Omega$, which implies that $x_{0} \in \Omega$. Let $\epsilon=\epsilon_{x_{0}}$ and $n=n_{x_{0}} \in S^{n-1}$ be the quantities which appear in (F2). It is not difficult to verify that (F2) implies

$$
f^{*}(y+r d)-f_{*}(y) \leqslant \omega\left(\left|y-x_{0}\right|+r\right)
$$

for all $y \in \Omega, r>0$ and $d \in S^{n-1},|d-n|<\epsilon$. 
Let us define for $\lambda>0, L \geqslant 1$ the function $\Phi: \bar{\Omega} \times \bar{\Omega} \rightarrow \mathbb{R}$ by

$$
\Phi(x, y):=u_{\theta}(x)-v(y)-L \lambda\left|x-y-\frac{1}{\lambda} n\right|^{2}-\left|x-x_{0}\right|^{2} .
$$

Let $\left(x_{\lambda}, y_{\lambda}\right) \in \bar{\Omega} \times \bar{\Omega}$ be such that

$$
\Phi\left(x_{\lambda}, y_{\lambda}\right)=\max _{(x, y) \in \bar{\Omega} \times \bar{\Omega}} \Phi(x, y) .
$$

Since $x_{0}-\frac{1}{\lambda} n \in \Omega$ for large $\lambda$, the relation $\Phi\left(x_{\lambda}, y_{\lambda}\right) \geqslant \Phi\left(x_{0}, x_{0}-\frac{1}{\lambda} n\right)$ implies together with 2.6 that

$$
\begin{aligned}
& L \lambda\left|x_{\lambda}-y_{\lambda}-\frac{1}{\lambda} n\right|^{2}+\left|x_{\lambda}-x_{0}\right|^{2} \leqslant u_{\theta}\left(x_{\lambda}\right)-v\left(y_{\lambda}\right)-u_{\theta}\left(x_{0}\right)+v\left(x_{0}-\frac{1}{\lambda} n\right) \\
& \quad=\left(u_{\theta}\left(x_{\lambda}\right)-v\left(x_{\lambda}\right)\right)-\left(u_{\theta}\left(x_{0}\right)-v\left(x_{0}\right)\right)+v\left(x_{\lambda}\right)-v\left(y_{\lambda}\right)-v\left(x_{0}\right)+v\left(x_{0}-\frac{1}{\lambda} n\right) \\
& \leqslant \operatorname{lip}(v)\left|x_{\lambda}-y_{\lambda}\right|+\operatorname{lip}(v) \frac{1}{\lambda} \\
& \leqslant \operatorname{lip}(v)\left|x_{\lambda}-y_{\lambda}-\frac{1}{\lambda} n\right|+2 \operatorname{lip}(v) \frac{1}{\lambda},
\end{aligned}
$$

where $\operatorname{lip}(v)$ denotes the Lipschitz constant of $v$. Therefore,

$$
L \lambda\left|x_{\lambda}-y_{\lambda}-\frac{1}{\lambda} n\right|^{2}+\left|x_{\lambda}-x_{0}\right|^{2} \leqslant C(\operatorname{lip}(v)) \frac{1}{\lambda},
$$

so that

$$
\begin{gathered}
x_{\lambda}, y_{\lambda} \rightarrow x_{0} \quad \text { as } \lambda \rightarrow \infty, \\
\lambda\left|x_{\lambda}-y_{\lambda}-\frac{1}{\lambda} n\right| \leqslant \frac{C}{\sqrt{L}}<\frac{\epsilon}{2+\epsilon}
\end{gathered}
$$

provided that $L$ is sufficiently large. Next, 2.8 implies that $u-\frac{1}{\theta} \zeta$ has a local maximum at $x_{\lambda}$ where $\zeta(x)=\tilde{v}\left(y_{\lambda}\right)+L \lambda\left|x-y_{\lambda}-\frac{1}{\lambda} n\right|^{2}+\left|x-x_{0}\right|^{2}$. Therefore,

$$
H\left(\frac{1}{\theta}\left(2 L \lambda\left(x_{\lambda}-y_{\lambda}-\frac{1}{\lambda} n\right)+2\left(x_{\lambda}-x_{0}\right)\right)\right) \leqslant f^{*}\left(x_{\lambda}\right),
$$

and similarly,

$$
H\left(2 L \lambda\left(x_{\lambda}-y_{\lambda}-\frac{1}{\lambda} n\right)\right) \geqslant f_{*}\left(y_{\lambda}\right) .
$$

Combining 2.12 and 2.13) and using assumptions (H1), (H2) we obtain

$$
\begin{aligned}
& f_{*}\left(y_{\lambda}\right) \leqslant \theta H\left(\frac{1}{\theta} 2 L \lambda\left(x_{\lambda}-y_{\lambda}-\frac{1}{\lambda} n\right)\right) \\
& \leqslant \theta\left(H\left(\frac{1}{\theta} 2 L \lambda\left(x_{\lambda}-y_{\lambda}-\frac{1}{\lambda} n\right)\right)-H\left(\frac{1}{\theta}\left(2 L \lambda\left(x_{\lambda}-y_{\lambda}-\frac{1}{\lambda} n\right)+2\left(x_{\lambda}-x_{0}\right)\right)\right)\right)+\theta f^{*}\left(x_{\lambda}\right) .
\end{aligned}
$$


Note that $H$ is locally Lipschitz continuous (since it is convex) so that we may deduce from 2.11 $\left.\mid H\left(\frac{1}{\theta} 2 L \lambda\left(x_{\lambda}-y_{\lambda}-\frac{1}{\lambda} n\right)\right)-H\left(\frac{1}{\theta}\left(2 L \lambda\left(x_{\lambda}-y_{\lambda}-\frac{1}{\lambda} n\right)+2\left(x_{\lambda}-x_{0}\right)\right)\right)\right)|\leqslant C| x_{\lambda}-x_{0} \mid$.

Inserting this inequality into (2.14) we arrive at

$$
(1-\theta) f_{*}\left(y_{\lambda}\right) \leqslant C\left|x_{\lambda}-x_{0}\right|+\theta\left(f^{*}\left(x_{\lambda}\right)-f_{*}\left(y_{\lambda}\right)\right) .
$$

In order to treat the second term we write $x_{\lambda}=y_{\lambda}+r_{\lambda} d_{\lambda}$ with

$$
d_{\lambda}=\frac{n+w_{\lambda}}{\left|n+w_{\lambda}\right|}, \quad r_{\lambda}=\frac{1}{\lambda}\left|n+w_{\lambda}\right|, \quad w_{\lambda}=\lambda\left(x_{\lambda}-y_{\lambda}-\frac{1}{\lambda} n\right) .
$$

Now, 2.11 implies

$$
\left|d_{\lambda}-n\right| \leqslant \frac{2\left|w_{\lambda}\right|}{1-\left|w_{\lambda}\right|} \leqslant \frac{\frac{2 \epsilon}{2+\epsilon}}{1-\frac{\epsilon}{2+\epsilon}}=\epsilon
$$

so that 2.77 yields

$$
f^{*}\left(x_{\lambda}\right)-f_{*}\left(y_{\lambda}\right)=f^{*}\left(y_{\lambda}+r_{\lambda} d_{\lambda}\right)-f_{*}\left(y_{\lambda}\right) \leqslant \omega\left(\left|y_{\lambda}-x_{0}\right|+r_{\lambda}\right) .
$$

If we insert this estimate into 2.15 and recall 2.1) the result is

$$
m(1-\theta) \leqslant C\left|x_{\lambda}-x_{0}\right|+\omega\left(\left|y_{\lambda}-x_{0}\right|+r_{\lambda}\right) .
$$

Sending $\lambda \nearrow \infty$ yields $m(1-\theta) \leqslant 0$ in view of 2.10, which is a contradiction. Thus, $u_{\theta} \leqslant v$ for all $\theta<1$ and sending $\theta \nearrow 1$ finally yields the result.

\section{Numerical scheme and error analysis}

Numerical schemes on uniform grids for Hamilton-Jacobi equations have been developed on the basis of an upwind discretisation of the gradient; see [11], [14] for an outline of the underlying ideas. It is possible to derive these schemes as well as methods on unstructured grids by interpreting the corresponding viscosity solution as the value function of an optimal control problem and by using the dynamic programming principle. We refer to Appendix A, written by M. Falcone, in [1] for a description of basic results together with a comprehensive list of references.

There is a close connection between the static Hamilton-Jacobi equation (1.1) and timedependent problems of the form

$$
u_{t}+H(\nabla u)=0 .
$$

Error estimates for finite difference approximations of [3.1] have been proved in [6], while [7] introduces a class of semi-Lagrangian schemes which are strongly related to Godunov methods in one space dimension.

We shall start from a class of finite difference schemes which are monotone and consistent and which satisfy a suitable stability condition. In order to keep the presentation simple we shall from now on assume that $\Omega=\prod_{i=1}^{n}\left(0, b_{i}\right)$. Let $h>0$ be such that there exist $N_{i} \in \mathbb{N}$ with $b_{i}=N_{i} h, i=1, \ldots, n$, and define

$$
\Omega_{h}:=\mathbb{Z}_{h}^{n} \cap \Omega, \quad \partial \Omega_{h}:=\mathbb{Z}_{h}^{n} \cap \partial \Omega, \quad \bar{\Omega}_{h}:=\Omega_{h} \cup \partial \Omega_{h},
$$


where $\mathbb{Z}_{h}^{n}=\left\{x_{\alpha}=\left(h \alpha_{1}, \ldots, h \alpha_{n}\right) \mid \alpha_{i} \in \mathbb{Z}, i=1, \ldots, n\right\}$. We shall approximate the viscosity solution $u$ by a grid function $U: \bar{\Omega}_{h} \rightarrow \mathbb{R}, U_{\alpha}=U\left(x_{\alpha}\right), \alpha=\left(\alpha_{1}, \ldots, \alpha_{n}\right) \in \mathbb{Z}^{n}$. For $x_{\alpha} \in \Omega_{h}$ and $k \in\{1, \ldots, n\}$ let

$$
D_{k}^{-} U_{\alpha}:=\frac{U_{\alpha}-U_{\alpha-e_{k}}}{h}, \quad D_{k}^{+} U_{\alpha}:=\frac{U_{\alpha+e_{k}}-U_{\alpha}}{h}
$$

be the usual backward and forward difference quotients. The numerical scheme now reads: find $U: \bar{\Omega}_{h} \rightarrow \mathbb{R}$ such that

$$
\begin{aligned}
H_{N}\left(D_{1}^{-} U_{\alpha}, D_{1}^{+} U_{\alpha}, \ldots, D_{n}^{-} U_{\alpha}, D_{n}^{+} U_{\alpha}\right) & =f\left(x_{\alpha}\right), & & x_{\alpha} \in \Omega_{h}, \\
U_{\alpha} & =\phi\left(x_{\alpha}\right), & & x_{\alpha} \in \partial \Omega_{h},
\end{aligned}
$$

where $H_{N}: \mathbb{R}^{2 n} \rightarrow \mathbb{R},\left(p_{1}, q_{1}, \ldots, p_{n}, q_{n}\right) \mapsto H_{N}\left(p_{1}, q_{1}, \ldots, p_{n}, q_{n}\right)$ is the numerical Hamiltonian. It is convenient to also introduce $F_{N}: \mathbb{R}^{2 n+1} \rightarrow \mathbb{R}, a=\left(a_{0}, a_{1}, a_{2}, \ldots, a_{2 n-1}, a_{2 n}\right) \mapsto$ $F_{N}(a)$, as

$$
F_{N}(a):=H_{N}\left(a_{0}-a_{1}, a_{2}-a_{0}, \ldots, a_{0}-a_{2 n-1}, a_{2 n}-a_{0}\right) .
$$

In what follows we shall assume that $H_{N}$ is locally Lipschitz continuous and has the following properties:

(HN1) Consistency:

$$
H_{N}\left(p_{1}, p_{1}, \ldots, p_{n}, p_{n}\right)=H\left(p_{1}, \ldots, p_{n}\right) \quad \text { for all } p=\left(p_{1}, \ldots, p_{n}\right) \in \mathbb{R}^{n} .
$$

(HN2) Monotonicity:

$$
\begin{aligned}
& a_{0} \mapsto F_{N}(a) \text { is increasing, } \\
& a_{k} \mapsto F_{N}(a) \text { is decreasing for } k=1, \ldots, 2 n .
\end{aligned}
$$

(HN3) Stability: there exists a function $Z: \bar{\Omega}_{h} \rightarrow \mathbb{R}$ which satisfies

$$
\begin{aligned}
H_{N}\left(D_{1}^{-} Z_{\alpha}, D_{1}^{+} Z_{\alpha}, \ldots, D_{n}^{-} Z_{\alpha}, D_{n}^{+} Z_{\alpha}\right) & \geqslant f\left(x_{\alpha}\right), & & x_{\alpha} \in \Omega_{h}, \\
Z_{\alpha} & =\phi\left(x_{\alpha}\right), & & x_{\alpha} \in \partial \Omega_{h}, \\
\left|D_{k}^{-} Z_{\alpha}\right|,\left|D_{k}^{+} Z_{\alpha}\right| & \leqslant R, & & x_{\alpha} \in \Omega_{h},
\end{aligned}
$$

where $R$ is independent of $h$.

The function $Z$ which appears in (HN3) will act as a discrete supersolution for solutions of (3.2), 3.3. We shall examine some examples of choices of $H_{N}$ in $\S 4$.

REMARK 3.1 Note that the function $Z$ in (HN3) above satisfies

$$
Z_{\alpha} \geqslant \phi_{\min }:=\min _{x \in \partial \Omega} \phi(x), \quad x_{\alpha} \in \bar{\Omega}_{h} .
$$

To see this, let $Z_{\beta}=\min _{x_{\alpha} \in \bar{\Omega}_{h}} Z_{\alpha}$ and assume that $x_{\beta} \in \Omega_{h}$. Then, 2.1, 3.8, and 3.7 would imply

$$
\begin{aligned}
m & \leqslant f\left(x_{\beta}\right) \leqslant H_{N}\left(D_{1}^{-} Z_{\beta}, D_{1}^{+} Z_{\beta}, \ldots, D_{n}^{-} Z_{\beta}, D_{n}^{+} Z_{\beta}\right) \\
& =F_{N}\left(\frac{Z_{\beta}}{h}, \frac{Z_{\beta-e_{1}}}{h}, \frac{Z_{\beta+e_{1}}}{h}, \ldots, \frac{Z_{\beta-e_{n}}}{h}, \frac{Z_{\beta+e_{n}}}{h}\right) \leqslant F_{N}\left(\frac{Z_{\beta}}{h}, \frac{Z_{\beta}}{h}, \frac{Z_{\beta}}{h}, \ldots, \frac{Z_{\beta}}{h}, \frac{Z_{\beta}}{h}\right) \\
& =H_{N}(0, \ldots, 0)=H(0)=0
\end{aligned}
$$

in view of (H1), a contradiction. Thus, $x_{\beta} \in \partial \Omega_{h}$ and 3.11 follows. 
Next, let us prove an auxiliary result which yields a kind of diagonal coercivity for the numerical Hamiltonian.

LEMMA 3.2 For $\left(a_{1}, a_{2}, \ldots, a_{2 n-1}, a_{2 n}\right) \in \mathbb{R}^{2 n}$,

$$
\lim _{t \rightarrow \infty} F_{N}\left(t, a_{1}, a_{2}, \ldots, a_{2 n-1}, a_{2 n}\right)=\infty \text {. }
$$

Proof. Using the defintion of $F_{N}$ along with 3.5 - 3.7) we deduce for $t \geqslant r:=\max \left(a_{1}, a_{2}, \ldots\right.$, $\left.a_{2 n-1}, a_{2 n}\right)$ that

$$
\begin{aligned}
F_{N}\left(t, a_{1}, a_{2}, \ldots, a_{2 n-1}, a_{2 n}\right) & \geqslant F_{N}(t, r, r, \ldots, r, r)=H_{N}(t-r, r-t, \ldots, t-r, r-t) \\
& =F_{N}(t-r, 0,0, \ldots, 0,0) \\
& \geqslant F_{N}(t-r, 0,2(t-r), \ldots, 0,2(t-r)) \\
& =H_{N}(t-r, t-r, \ldots, t-r, t-r)=H(t-r, \ldots, t-r) \\
& \rightarrow \infty \quad \text { as } t \rightarrow \infty,
\end{aligned}
$$

which proves the lemma.

Lemma 3.3 Assume that (HN1)-(HN3) and (F1) hold. Then there exists a solution $U$ of (3.2), 3.3, which satisfies $\phi_{\min } \leqslant U_{\alpha} \leqslant Z_{\alpha}$ for all $x_{\alpha} \in \bar{\Omega}_{h}$.

Proof. We consider the following iteration: let $U^{0}:=Z$ and given $U^{k}: \bar{\Omega}_{h} \rightarrow \mathbb{R}$, let

$$
\begin{aligned}
& U_{\alpha}^{k+1}=\inf \left\{t \mid F_{N}\left(\frac{t}{h}, \frac{U_{\alpha-e_{1}}^{k}}{h}, \frac{U_{\alpha+e_{1}}^{k}}{h}, \ldots, \frac{U_{\alpha-e_{n}}^{k}}{h}, \frac{U_{\alpha+e_{n}}^{k}}{h}\right) \geqslant f\left(x_{\alpha}\right)\right\}, \quad x_{\alpha} \in \Omega_{h}, \\
& U_{\alpha}^{k+1}=\phi\left(x_{\alpha}\right), \quad x_{\alpha} \in \partial \Omega_{h} .
\end{aligned}
$$

We claim that the sequence $\left(U^{k}\right)_{k \in \mathbb{N}}$ is well defined and that

$$
\phi_{\min } \leqslant U^{k} \leqslant U^{k-1} \leqslant Z \text { for all } k \in \mathbb{N} \text {. }
$$

To see this, assume that 3.12 holds for all $1 \leqslant j \leqslant k$ and consider, for $x_{\alpha} \in \Omega_{h}$,

$$
\eta(t):=F_{N}\left(\frac{t}{h}, \frac{U_{\alpha-e_{1}}^{k}}{h}, \frac{U_{\alpha+e_{1}}^{k}}{h}, \ldots, \frac{U_{\alpha-e_{n}}^{k}}{h}, \frac{U_{\alpha+e_{n}}^{k}}{h}\right), \quad t \in \mathbb{R} .
$$

Clearly, $\eta$ is continuous and increasing. Lemma 3.2 implies that $\eta(t) \rightarrow \infty$ as $t \rightarrow \infty$ so that $U_{\alpha}^{k+1}$ is well defined. Since $U^{k} \geqslant \phi_{\min }$ by our induction hypothesis, 3.7, 3.5 and (H1) yield

$$
\begin{aligned}
\eta\left(\phi_{\min }\right) & =F_{N}\left(\frac{\phi_{\min }}{h}, \frac{U_{\alpha-e_{1}}^{k}}{h}, \frac{U_{\alpha+e_{1}}^{k}}{h}, \ldots, \frac{U_{\alpha-e_{n}}^{k}}{h}, \frac{U_{\alpha+e_{n}}^{k}}{h}\right) \\
& \leqslant F_{N}\left(\frac{\phi_{\min }}{h}, \ldots, \frac{\phi_{\min }}{h}\right)=H(0)=0<f\left(x_{\alpha}\right),
\end{aligned}
$$

which implies that $U_{\alpha}^{k+1} \geqslant \phi_{\min }$. Also, as $U^{k} \leqslant U^{k-1}, 3.7$ yields

$$
\begin{aligned}
\phi\left(U_{\alpha}^{k}\right) & =F_{N}\left(\frac{U_{\alpha}^{k}}{h}, \frac{U_{\alpha-e_{1}}^{k}}{h}, \frac{U_{\alpha+e_{1}}^{k}}{h}, \ldots, \frac{U_{\alpha-e_{n}}^{k}}{h}, \frac{U_{\alpha+e_{n}}^{k}}{h}\right) \\
& \geqslant F_{N}\left(\frac{U_{\alpha}^{k}}{h}, \frac{U_{\alpha-e_{1}}^{k-1}}{h}, \frac{U_{\alpha+e_{1}}^{k-1}}{h}, \ldots, \frac{U_{\alpha-e_{n}}^{k-1}}{h}, \frac{U_{\alpha+e_{n}}^{k-1}}{h}\right) \geqslant f\left(x_{\alpha}\right)
\end{aligned}
$$


by the definition of $U_{\alpha}^{k}$. Thus $U_{\alpha}^{k+1} \leqslant U_{\alpha}^{k}$. Using similar arguments and recalling (3.11) we infer that 3.12 holds for $k=1$, so that we finally obtain $(3.12)$ for all $k \in \mathbb{N}$. Note also that

$$
F_{N}\left(\frac{U_{\alpha}^{k+1}}{h}, \frac{U_{\alpha-e_{1}}^{k}}{h}, \frac{U_{\alpha+e_{1}}^{k}}{h}, \ldots, \frac{U_{\alpha-e_{n}}^{k}}{h}, \frac{U_{\alpha+e_{n}}^{k}}{h}\right)=f\left(x_{\alpha}\right), \quad x_{\alpha} \in \Omega_{h}, k \in \mathbb{N} .
$$

From 3.12 we infer that $U_{\alpha}^{k} \rightarrow U_{\alpha}$ for all $x_{\alpha} \in \bar{\Omega}_{h}$ as $k \rightarrow \infty$. Clearly, $U_{\alpha}=\phi\left(x_{\alpha}\right)$ for $x_{\alpha} \in \partial \Omega_{h}$. Letting $k \rightarrow \infty$ in (3.14) and using the continuity of $F_{N}$ finally implies that $U$ satisfies (3.2). The bounds on $U$ follow from 3.12 .

Our main result of this paragraph is an error bound between a discrete solution $U$ and the viscosity solution $u$. It is natural to model the error analysis on the uniqueness proof, but in our case a closer inspection of the proof of Theorem 2.3 shows that it is not obvious how to control the difference between the viscosity solution $u$ and an approximation $U$. Therefore we recall a different approach to prove uniqueness, namely to apply the Kruzhkov transform. The advantage is that the equation satisfied by the transformed function contains an additional term of zero order (cf. (3.15)), which is subsequently exploited.

TheOREM 3.4 Assume that (F1), (F2)*, (C) as well as (HN1)-(HN3) hold. Let $u$ be the viscosity solution of (1.1), (1.2) and $U$ a solution of (3.2), (3.3) which satisfies $\phi_{\min } \leqslant U \leqslant Z$. Then there exists a constant $C$, which is independent of $h$, such that

$$
\max _{x_{\alpha} \in \bar{\Omega}_{h}}\left|u\left(x_{\alpha}\right)-U\left(x_{\alpha}\right)\right| \leqslant C \sqrt{h} .
$$

Proof. As mentioned above we introduce the Kruzhkov transform of $u$ and $U$, i.e. $\tilde{u}: \bar{\Omega} \rightarrow \mathbb{R}$, $\tilde{U}: \bar{\Omega}_{h} \rightarrow \mathbb{R}$ which are defined by

$$
\tilde{u}(x):=-e^{-u(x)}, x \in \bar{\Omega}, \quad \tilde{U}_{\alpha}:=-e^{-U_{\alpha}}, x_{\alpha} \in \bar{\Omega}_{h} .
$$

Clearly, $\tilde{u}(x)=-e^{-\phi(x)}$ for $x \in \partial \Omega$ and one verifies (cf. [5]) that $\tilde{u}$ is a viscosity supersolution of

$$
f(x) \tilde{u}-\tilde{u} H\left(-\frac{1}{\tilde{u}} \nabla \tilde{u}\right)=0
$$

in the sense that if $\zeta \in C^{\infty}(\Omega)$ and $\tilde{u}-\zeta$ has a local minimum at a point $x_{0} \in \Omega$, then

$$
f_{*}\left(x_{0}\right) \tilde{u}\left(x_{0}\right)-\tilde{u}\left(x_{0}\right) H\left(-\frac{1}{\tilde{u}\left(x_{0}\right)} \nabla \zeta\left(x_{0}\right)\right) \geqslant 0 .
$$

Note also that

$$
\tilde{H}(x, r, p):=f(x) r-r H\left(-\frac{1}{r} p\right), \quad(x, r, p) \in \Omega \times \mathbb{R} \backslash\{0\} \times \mathbb{R}^{n},
$$

satisfies in view of 2.1] and the convexity of $H$ (cf. [5])

$$
\frac{\partial \tilde{H}}{\partial r}(x, r, p)=f(x)-H\left(-\frac{1}{r} p\right)+\left(D H\left(-\frac{1}{r} p\right),-\frac{1}{r} p\right) \geqslant f(x) \geqslant m
$$

uniformly in $(x, r, p)$. 
Next, let $x_{\beta} \in \bar{\Omega}_{h}$ be such that

$$
\left|\tilde{u}\left(x_{\beta}\right)-\tilde{U}_{\beta}\right|=\max _{x_{\alpha} \in \bar{\Omega}_{h}}\left|\tilde{u}\left(x_{\alpha}\right)-\tilde{U}_{\alpha}\right|
$$

and assume that $\tilde{U}_{\beta} \geqslant \tilde{u}\left(x_{\beta}\right)$, the case $\tilde{u}\left(x_{\beta}\right)>\tilde{U}_{\beta}$ being treated in a similar way.

Let us first consider the situation when

$$
\left(x_{\beta}\right)_{i} \leqslant \sqrt{h} \quad \text { or } \quad\left(x_{\beta}\right)_{i} \geqslant b_{i}-\sqrt{h} \quad \text { for some } i \in\{1, \ldots, n\} .
$$

In the first case, let $x_{\beta_{0}}=\left(\beta_{1} h, \ldots, \beta_{i-1} h, 0, \beta_{i+1} h, \ldots, \beta_{n} h\right) \in \partial \Omega$ and $\tilde{Z}_{\alpha}:=-e^{-Z_{\alpha}}$. Since $\tilde{u}\left(x_{\beta_{0}}\right)=-e^{-\phi\left(x_{\beta_{0}}\right)}=\tilde{Z}_{\beta_{0}}$ we deduce with the help of 3.10 that

$$
\begin{aligned}
\tilde{U}_{\beta}-\tilde{u}\left(x_{\beta}\right) & =\left(\tilde{U}_{\beta}-\tilde{u}\left(x_{\beta_{0}}\right)\right)+\left(\tilde{u}\left(x_{\beta_{0}}\right)-\tilde{u}\left(x_{\beta}\right)\right) \leqslant\left(\tilde{Z}_{\beta}-\tilde{Z}_{\beta_{0}}\right)+\left(\tilde{u}\left(x_{\beta_{0}}\right)-\tilde{u}\left(x_{\beta}\right)\right) \\
& \leqslant(C(R)+\operatorname{lip}(\tilde{u}))\left|x_{\beta}-x_{\beta_{0}}\right| \leqslant(C(R)+\operatorname{lip}(\tilde{u})) \sqrt{h} .
\end{aligned}
$$

Arguing in a similar way if $\left(x_{\beta}\right)_{i} \geqslant b_{i}-\sqrt{h}$ we conclude that

$$
\max _{x_{\alpha} \in \bar{\Omega}_{h}}\left|\tilde{u}\left(x_{\alpha}\right)-\tilde{U}_{\alpha}\right|=\tilde{U}_{\beta}-\tilde{u}\left(x_{\beta}\right) \leqslant C \sqrt{h}
$$

if 3.18 holds. Now we consider the case

$$
\sqrt{h}<\left(x_{\beta}\right)_{i}<b_{i}-\sqrt{h} \quad \text { for } i=1, \ldots, n .
$$

Let $\epsilon>0, K \geqslant 0, n=n_{x_{\beta}}$ be the quantities appearing in 2.3 and define $\Phi: \bar{\Omega} \times \bar{\Omega}_{h} \rightarrow \mathbb{R}$ by

$$
\Phi\left(x, x_{\alpha}\right):=\tilde{U}_{\alpha}-\tilde{u}(x)-\frac{L_{1}}{\sqrt{h}}\left|x_{\alpha}-x-\sqrt{h} n\right|^{2}-L_{2} \sqrt{h}\left|x_{\alpha}-x_{\beta}\right|^{2},
$$

where $L_{1}, L_{2} \geqslant 0$ are constants that do not depend on $h$ and which will be chosen later. There exists $\left(x_{h}, x_{\alpha_{h}}\right) \in \bar{\Omega} \times \bar{\Omega}_{h}$ such that

$$
\Phi\left(x_{h}, x_{\alpha_{h}}\right)=\max _{\left(x, x_{\alpha}\right) \in \bar{\Omega} \times \bar{\Omega}_{h}} \Phi\left(x, x_{\alpha}\right) .
$$

In view of 3.20 we have $x_{\beta}-\sqrt{h} n \in \bar{\Omega}$ and therefore

$$
\Phi\left(x_{h}, x_{\alpha_{h}}\right) \geqslant \Phi\left(x_{\beta}-\sqrt{h} n, x_{\beta}\right)
$$

or equivalently

$$
\tilde{U}_{\alpha_{h}}-\tilde{u}\left(x_{h}\right)-\frac{L_{1}}{\sqrt{h}}\left|x_{\alpha_{h}}-x_{h}-\sqrt{h} n\right|^{2}-L_{2} \sqrt{h}\left|x_{\alpha_{h}}-x_{\beta}\right|^{2} \geqslant \tilde{U}_{\beta}-\tilde{u}\left(x_{\beta}-\sqrt{h} n\right) .
$$


This implies

$$
\begin{aligned}
\frac{L_{1}}{\sqrt{h}} \mid x_{\alpha_{h}} & -x_{h}-\left.\sqrt{h} n\right|^{2}+L_{2} \sqrt{h}\left|x_{\alpha_{h}}-x_{\beta}\right|^{2} \\
& \leqslant \tilde{u}\left(x_{\beta}-\sqrt{h} n\right)-\tilde{u}\left(x_{h}\right)+\tilde{U}_{\alpha_{h}}-\tilde{U}_{\beta} \\
& \leqslant \tilde{u}\left(x_{\alpha_{h}}\right)-\tilde{u}\left(x_{h}\right)+\left(\left(\tilde{U}_{\alpha_{h}}-\tilde{u}\left(x_{\alpha_{h}}\right)\right)-\left(\tilde{U}_{\beta}-\tilde{u}\left(x_{\beta}\right)\right)\right)+\tilde{u}\left(x_{\beta}-\sqrt{h} n\right)-\tilde{u}\left(x_{\beta}\right) \\
& \leqslant \operatorname{lip}(\tilde{u})\left|x_{\alpha_{h}}-x_{h}\right|+\sqrt{h} \operatorname{lip}(\tilde{u}) \\
& \leqslant \operatorname{lip}(\tilde{u})\left|x_{\alpha_{h}}-x_{h}-\sqrt{h} n\right|+2 \sqrt{h} \operatorname{lip}(\tilde{u}) \\
& \leqslant \frac{L_{1}}{2 \sqrt{h}}\left|x_{\alpha_{h}}-x_{h}-\sqrt{h} n\right|^{2}+\frac{\sqrt{h}}{2 L_{1}} \operatorname{lip}(\tilde{u})^{2}+2 \sqrt{h} \operatorname{lip}(\tilde{u}),
\end{aligned}
$$

and therefore,

$$
\begin{gathered}
\frac{1}{h}\left|x_{\alpha_{h}}-x_{h}-\sqrt{h} n\right|^{2} \leqslant \frac{1}{L_{1}^{2}} \operatorname{lip}(\tilde{u})^{2}+\frac{4}{L_{1}} \operatorname{lip}(\tilde{u})<\left(\frac{\epsilon}{2+\epsilon}\right)^{2} \\
\left|x_{\alpha_{h}}-x_{\beta}\right|^{2} \leqslant \frac{1}{2 L_{1} L_{2}} \operatorname{lip}(\tilde{u})^{2}+\frac{2}{L_{2}} \operatorname{lip}(\tilde{u})<\epsilon^{2}
\end{gathered}
$$

provided that $L_{1}, L_{2}$ are sufficiently large.

Let us first consider the case that $\left(x_{h}, x_{\alpha_{h}}\right) \in \Omega \times \Omega_{h}$. We infer from 3.16) that

$$
f_{*}\left(x_{h}\right) \tilde{u}\left(x_{h}\right)-\tilde{u}\left(x_{h}\right) H\left(-\frac{1}{\tilde{u}\left(x_{h}\right)} \frac{2 L_{1}}{\sqrt{h}}\left(x_{\alpha_{h}}-x_{h}-\sqrt{h} n\right)\right) \geqslant 0 .
$$

In order to derive a corresponding relation for the discrete solution, we consider the inequality $\Phi\left(x_{h}, x_{\alpha_{h}}\right) \geqslant \Phi\left(x_{h}, x_{\alpha}\right)$ for all $x_{\alpha} \in \bar{\Omega}$, which translates into

$$
\begin{aligned}
\tilde{U}_{\alpha} \leqslant & \tilde{U}_{\alpha_{h}}+\frac{L_{1}}{\sqrt{h}}\left(\left|x_{\alpha}-x_{h}-\sqrt{h} n\right|^{2}-\left|x_{\alpha_{h}}-x_{h}-\sqrt{h} n\right|^{2}\right) \\
& \quad+L_{2} \sqrt{h}\left(\left|x_{\alpha}-x_{\beta}\right|^{2}-\left|x_{\alpha_{h}}-x_{\beta}\right|^{2}\right) \\
=: & \tilde{V}_{\alpha} .
\end{aligned}
$$

Note first that

$$
\begin{aligned}
& D_{k}^{-} \tilde{V}_{\alpha}=\frac{2 L_{1}}{\sqrt{h}}\left(x_{\alpha}-x_{h}-\sqrt{h} n, e_{k}\right)+2 L_{2} \sqrt{h}\left(x_{\alpha}-x_{\beta}, e_{k}\right)-L_{1} \sqrt{h}-L_{2} h^{3 / 2}, \\
& D_{k}^{+} \tilde{V}_{\alpha}=\frac{2 L_{1}}{\sqrt{h}}\left(x_{\alpha}-x_{h}-\sqrt{h} n, e_{k}\right)+2 L_{2} \sqrt{h}\left(x_{\alpha}-x_{\beta}, e_{k}\right)+L_{1} \sqrt{h}+L_{2} h^{3 / 2},
\end{aligned}
$$

and therefore by 3.22,

$$
\begin{aligned}
& \left|D_{k}^{-} \tilde{V}_{\alpha_{h}}\right|,\left|D_{k}^{-} \tilde{V}_{\alpha_{h}}\right| \leqslant C \\
& \left|D_{k}^{ \pm} \tilde{V}_{\alpha_{h}}-\frac{2 L_{1}}{\sqrt{h}}\left(x_{\alpha_{h}}-x_{h}-\sqrt{h} n, e_{k}\right)\right| \leqslant C \sqrt{h}, \quad k=1, \ldots, n,
\end{aligned}
$$


uniformly in $h$. Recalling that $U \leqslant Z$ we also deduce from (3.9) and (3.10) that

$$
\tilde{V}_{\alpha_{h}}=\tilde{U}_{\alpha_{h}}=-e^{-U_{\alpha_{h}}} \leqslant-e^{-Z_{\alpha_{h}}} \leqslant-\bar{c},
$$

where $\bar{c}>0$ depends on $\phi$ and $R$. Furthermore, 3.26 implies

$$
\tilde{V}_{\alpha_{h} \pm e_{k}} \leqslant-\frac{1}{2} \bar{c} \quad \text { for } h \text { sufficiently small. }
$$

Thus we can define $V_{\alpha}:=-\log \left(-\tilde{V}_{\alpha}\right)$ for $\alpha=\alpha_{h}, \alpha_{h} \pm e_{k}$ and the mean value theorem yields

$$
D_{k}^{-} \tilde{V}_{\alpha_{h}}=e^{-\xi_{k}^{-}} D_{k}^{-} V_{\alpha_{h}}, \quad D_{k}^{+} \tilde{V}_{\alpha}=e^{-\xi_{k}^{+}} D_{k}^{+} V_{\alpha}, \quad k=1, \ldots, n,
$$

where $\xi_{k}^{-}$lies between $V_{\alpha}, V_{\alpha-e_{k}}$ and $\xi_{k}^{+}$lies between $V_{\alpha}, V_{\alpha+e_{k}}$. In particular,

$$
e^{\xi_{k}^{ \pm}} \leqslant \max \left(e^{V_{\alpha_{h} \pm e_{k}}}, e^{V_{\alpha_{h}}}\right)=\max \left(\frac{-1}{\tilde{V}_{\alpha_{h} \pm e_{k}}}, \frac{-1}{\tilde{V}_{\alpha_{h}}}\right) \leqslant \frac{2}{\bar{c}}
$$

by 3.28. Thus, 3.29, 3.26, 3.22, 3.27) together with the fact that $\phi_{\min } \leqslant U \leqslant Z$ imply

$$
\left|D_{k}^{ \pm} V_{\alpha_{h}}\right| \leqslant C, \quad k=1, \ldots, n, \quad\left|-\frac{1}{\tilde{U}_{\alpha_{h}}} \frac{2 L_{1}}{\sqrt{h}}\left(x_{\alpha_{h}}-x_{h}-\sqrt{h} n\right)\right| \leqslant C .
$$

Next, we deduce from 3.25 that

$$
U_{\alpha_{h}}=V_{\alpha_{h}}, \quad U_{\alpha} \leqslant V_{\alpha}, \alpha=\alpha_{h} \pm e_{k}, k=1, \ldots, n,
$$

so that the monotonicity property 3.7 and 3.2 imply

$$
\begin{aligned}
f\left(x_{\alpha_{h}}\right) & =H_{N}\left(D_{1}^{-} U_{\alpha_{h}}, D_{1}^{+} U_{\alpha_{h}}, \ldots, D_{n}^{-} U_{\alpha_{h}}, D_{n}^{+} U_{\alpha_{h}}\right) \\
& \geqslant H_{N}\left(D_{1}^{-} V_{\alpha_{h}}, D_{1}^{+} V_{\alpha_{h}}, \ldots, D_{n}^{-} V_{\alpha_{h}}, D_{n}^{+} V_{\alpha_{h}}\right) .
\end{aligned}
$$

Multiplying the above inequality by $\tilde{U}_{\alpha_{h}}<0$, using 3.5 along with 3.31 and the local Lipschitz continuity of $H_{N}$ we infer

$$
\begin{aligned}
& f\left(x_{\alpha_{h}}\right) \tilde{U}_{\alpha_{h}}-\tilde{U}_{\alpha_{h}} H\left(-\frac{1}{\tilde{U}_{\alpha_{h}}} \frac{2 L_{1}}{\sqrt{h}}\left(x_{\alpha_{h}}-x_{h}-\sqrt{h} n\right)\right) \\
& \leqslant \tilde{U}_{\alpha_{h}}\left(H_{N}\left(D_{1}^{-} V_{\alpha_{h}}, D_{1}^{+} V_{\alpha_{h}}, \ldots, D_{n}^{-} V_{\alpha_{h}}, D_{n}^{+} V_{\alpha_{h}}\right)-H\left(-\frac{1}{\tilde{U}_{\alpha_{h}}} \frac{2 L_{1}}{\sqrt{h}}\left(x_{\alpha_{h}}-x_{h}-\sqrt{h} n\right)\right)\right) \\
& \leqslant C \max _{k=1, \ldots, n}\left|D_{k}^{ \pm} V_{\alpha_{h}}+\frac{1}{\tilde{U}_{\alpha_{h}}} \frac{2 L_{1}}{\sqrt{h}}\left(x_{\alpha_{h}}-x_{h}-\sqrt{h} n, e_{k}\right)\right| \\
& \leqslant C \max _{k=1, \ldots, n}\left(e^{\xi_{k}^{ \pm}}\left|D_{k}^{ \pm} \tilde{V}_{\alpha_{h}}-\frac{2 L_{1}}{\sqrt{h}}\left(x_{\alpha_{h}}-x_{h}-\sqrt{h} n, e_{k}\right)\right|\right. \\
& \left.\quad+\left|e^{\xi_{k}^{ \pm}}-e^{V_{\alpha_{h}}}\right| \frac{2 L_{1}}{\sqrt{h}}\left(x_{\alpha_{h}}-x_{h}-\sqrt{h} n, e_{k}\right) \mid\right) \\
& \leqslant C \sqrt{h}+C h .
\end{aligned}
$$


Note that the last estimate is a consequence of 3.26 and 3.30). If we combine 3.24) with 3.32 and use the definition of $\tilde{H}$ we obtain

$$
\begin{array}{r}
\tilde{H}\left(x_{\alpha_{h}}, \tilde{U}_{\alpha_{h}}, \frac{2 L_{1}}{\sqrt{h}}\left(x_{\alpha_{h}}-x_{h}-\sqrt{h} n\right)\right)-\tilde{H}\left(x_{\alpha_{h}}, \tilde{u}\left(x_{h}\right), \frac{2 L_{1}}{\sqrt{h}}\left(x_{\alpha_{h}}-x_{h}-\sqrt{h} n\right)\right) \\
\leqslant C \sqrt{h}+e^{-u\left(x_{h}\right)}\left(f\left(x_{\alpha_{h}}\right)-f_{*}\left(x_{h}\right)\right) .
\end{array}
$$

Note first that

$$
\begin{aligned}
\tilde{U}_{\alpha_{h}}-\tilde{u}\left(x_{h}\right) & =\Phi\left(x_{h}, x_{\alpha_{h}}\right)+\frac{L_{1}}{\sqrt{h}}\left|x_{\alpha_{h}}-x_{h}-\sqrt{h} n\right|^{2}+L_{2} \sqrt{h}\left|x_{\alpha_{h}}-x_{\beta}\right|^{2} \\
& \geqslant \Phi\left(x_{\beta}, x_{\beta}\right)=\tilde{U}_{\beta}-\tilde{u}\left(x_{\beta}\right)-L_{1} \sqrt{h} .
\end{aligned}
$$

Thus, in view of (3.17),

$$
m\left(\tilde{U}_{\beta}-\tilde{u}\left(x_{\beta}\right)\right) \leqslant e^{-u\left(x_{h}\right)}\left(f\left(x_{\alpha_{h}}\right)-f_{*}\left(x_{h}\right)\right)+C \sqrt{h} .
$$

Let us write $x_{\alpha_{h}}=x_{h}+r_{h} d_{h}$ with

$$
d_{h}=\frac{n+w_{h}}{\left|n+w_{h}\right|}, \quad r_{h}=\sqrt{h}\left|n+w_{h}\right|, \quad w_{h}=\frac{1}{\sqrt{h}}\left(x_{\alpha_{h}}-x_{h}-\sqrt{h} n\right) .
$$

Note that 3.23) implies that $\left|x_{\alpha_{h}}-x_{\beta}\right|<\epsilon$; 3.22) and a similar argument as in 2.16) yield $\left|d_{h}-n\right|<\epsilon$, so that 2.3 gives

$$
f\left(x_{\alpha_{h}}\right)-f_{*}\left(x_{h}\right) \leqslant f^{*}\left(x_{h}+r_{h} d_{h}\right)-f_{*}\left(x_{h}\right) \leqslant K r_{h} \leqslant C \sqrt{h} .
$$

Combining 3.33 and 3.34 finally yields

$$
\max _{x_{\alpha} \in \bar{\Omega}_{h}}\left|\tilde{u}\left(x_{\alpha}\right)-\tilde{U}_{\alpha}\right|=\tilde{U}_{\beta}-\tilde{u}\left(x_{\beta}\right) \leqslant C \sqrt{h} .
$$

It remains to consider the case when $x_{\alpha_{h}} \in \partial \Omega_{h}$ or $x_{h} \in \partial \Omega$. If $x_{\alpha_{h}} \in \partial \Omega_{h}$, it follows from 3.21, the fact that $\tilde{u}\left(x_{\alpha_{h}}\right)=\tilde{U}_{\alpha_{h}}$ and 3.22 that

$$
\begin{aligned}
\tilde{U}_{\beta}-\tilde{u}\left(x_{\beta}\right) & \leqslant \tilde{u}\left(x_{\beta}-\sqrt{h} n\right)-\tilde{u}\left(x_{\beta}\right)+\tilde{u}\left(x_{\alpha_{h}}\right)-\tilde{u}\left(x_{h}\right) \\
& \leqslant \operatorname{lip}(\tilde{u})\left(\sqrt{h}+\left|x_{\alpha_{h}}-x_{h}\right|\right) \\
& \leqslant \operatorname{lip}(\tilde{u})\left(2 \sqrt{h}+\left|x_{\alpha_{h}}-x_{h}-\sqrt{h} n\right|\right) \leqslant C \sqrt{h} .
\end{aligned}
$$

Let us finally assume that $x_{h} \in \partial \Omega$. Since $\tilde{u}\left(x_{h}\right)=\phi\left(x_{h}\right)=\tilde{Z}_{x_{h}}$ and $\tilde{U} \leqslant \tilde{Z}$ we obtain

$$
\begin{aligned}
\tilde{U}_{\beta}-\tilde{u}\left(x_{\beta}\right) & \leqslant \tilde{U}_{\alpha_{h}}-\tilde{u}\left(x_{h}\right)+\tilde{u}\left(x_{\beta}-\sqrt{h} n\right)-\tilde{u}\left(x_{\beta}\right) \\
& \leqslant \tilde{Z}\left(x_{\alpha_{h}}\right)-\tilde{Z}\left(x_{h}\right)+\tilde{u}\left(x_{\beta}-\sqrt{h} n\right)-\tilde{u}\left(x_{\beta}\right) \leqslant C \sqrt{h}
\end{aligned}
$$

as above. If we combine $3.19,3.35$ with the above estimates we obtain $\max _{x_{\alpha} \in \bar{\Omega}_{h}}\left|\tilde{u}\left(x_{\alpha}\right)-\tilde{U}_{\alpha}\right| \leqslant$ $C \sqrt{h}$. Transforming back to $u$ and $U$ implies the desired error bound. 


\section{Examples of numerical Hamiltonians}

Let us consider some examples of numerical Hamiltonians $H_{N}$. In order to simplify matters we restrict ourselves to the case of two space dimensions and a domain $\Omega$ of the form $\Omega=\left(0, b_{1}\right) \times$ $\left(0, b_{2}\right)$ with $b_{2} \leqslant b_{1}$.

\subsection{Viscous regularization}

Suppose that $H$ is globally Lipschitz continuous with Lipschitz constant $L$. We define $H_{N}: \mathbb{R}^{4} \rightarrow$ $\mathbb{R}$ by

$$
H_{N}\left(p_{1}, q_{1}, p_{2}, q_{2}\right):=-\frac{L}{2} \sum_{k=1}^{2}\left(q_{k}-p_{k}\right)+H\left(\frac{p_{1}+q_{1}}{2}, \frac{p_{2}+q_{2}}{2}\right)
$$

and verify (HN1)-(HN3).

(HN1) Clearly, $H_{N}\left(p_{1}, p_{1}, p_{2}, p_{2}\right)=H\left(p_{1}, p_{2}\right)$.

(HN2) The function $F_{N}$ defined in 3.4 is given by

$$
F_{N}\left(a_{0}, a_{1}, \ldots, a_{4}\right)=2 L a_{0}-\frac{L}{2} \sum_{k=1}^{2}\left(a_{2 k-1}+a_{2 k}\right)+H\left(\frac{a_{2}-a_{1}}{2}, \frac{a_{4}-a_{3}}{2}\right),
$$

and (3.6) is evident. We check (3.7) for $k=1$ : from the Lipschitz continuity of $H$ we deduce for $a_{1}<\tilde{a}_{1}$ that

$$
\begin{aligned}
F_{N}\left(a_{0}, a_{1}, a_{2}, a_{3}, a_{4}\right)- & F_{N}\left(a_{0}, \tilde{a}_{1}, a_{2}, a_{3}, a_{4}\right) \\
& =\frac{L}{2}\left(\tilde{a}_{1}-a_{1}\right)+H\left(\frac{a_{2}-a_{1}}{2}, \frac{a_{4}-a_{3}}{2}\right)-H\left(\frac{a_{2}-\tilde{a}_{1}}{2}, \frac{a_{4}-a_{3}}{2}\right) \\
& \geqslant \frac{L}{2}\left(\tilde{a}_{1}-a_{1}\right)-L\left|\frac{a_{2}-a_{1}}{2}-\frac{a_{2}-\tilde{a}_{1}}{2}\right|=0
\end{aligned}
$$

i.e. $a_{1} \mapsto F_{N}\left(a_{0}, a_{1}, a_{2}, a_{3}, a_{4}\right)$ is decreasing.

(HN3) Let us consider $d(x):=\operatorname{dist}(x, \partial \Omega), x=\left(x_{1}, x_{2}\right)$. It is not difficult to see that

$$
d(x)= \begin{cases}x_{1} & \text { in } \Omega_{1}=\left\{x \in \Omega \mid 0 \leqslant x_{2} \leqslant b_{2}, 0 \leqslant x_{1} \leqslant \min \left(b_{2}-x_{2}, x_{2}\right)\right\}, \\ b_{1}-x_{1} & \text { in } \Omega_{2}=\left\{x \in \Omega \mid 0 \leqslant x_{2} \leqslant b_{2}, b_{1} \geqslant x_{1} \geqslant \max \left(b_{1}-x_{2}, b_{1}-b_{2}+x_{2}\right)\right\}, \\ x_{2} & \text { in } \Omega_{3}=\left\{x \in \Omega \mid 0 \leqslant x_{1} \leqslant b_{1}, 0 \leqslant x_{2} \leqslant \min \left(x_{1}, b_{2} / 2, b_{1}-x_{1}\right)\right\}, \\ b_{2}-x_{2} & \text { in } \Omega_{4}=\left\{x \in \Omega \mid 0 \leqslant x_{1} \leqslant b_{1}, b_{2} \geqslant x_{2} \geqslant \max \left(b_{2}-x_{1}, b_{2} / 2, x_{1}+b_{2}-b_{1}\right)\right\} .\end{cases}
$$

We may assume that $\phi$ is defined as a Lipschitz continuous function on $\bar{\Omega}$ with Lipschitz constant $L_{\phi}$ (one such extension is e.g. given by the viscosity solution of $(1.1),(1.2)$ ).

Define $Z: \bar{\Omega}_{h} \rightarrow \mathbb{R}$ by $Z_{\alpha}:=\phi_{\alpha}+\rho d\left(x_{\alpha}\right)$, where $\phi_{\alpha}=\phi\left(x_{\alpha}\right)$ and $\rho$ is independent of $h$. Clearly, $Z$ satisfies (3.9) as well as (3.10) with $R=L_{\phi}+\rho$. We claim that (3.8) holds provided that $\rho$ is sufficiently large and verify this for a point $x_{\alpha}=\left(x_{1 \alpha}, x_{2 \alpha}\right) \in \Omega_{1}$. 
Case 1: $x_{2 \alpha}-h \leqslant x_{1 \alpha} \leqslant x_{2 \alpha}$. Since $x_{\alpha+e_{1}}, x_{\alpha-e_{2}} \in \Omega_{3}$ it follows that $d\left(x_{\alpha-e_{1}}\right)=x_{1 \alpha}-h$, $d\left(x_{\alpha+e_{1}}\right)=x_{2 \alpha}, d\left(x_{\alpha-e_{2}}\right)=x_{2 \alpha}-h, d\left(x_{\alpha+e_{2}}\right)=x_{1 \alpha}$ and therefore

$$
\begin{aligned}
& D_{1}^{-} Z_{\alpha}=D_{1}^{-} \phi_{\alpha}+\rho, \quad D_{1}^{+} Z_{\alpha}=D_{1}^{+} \phi_{\alpha}+\rho \frac{x_{2 \alpha}-x_{1 \alpha}}{h} \leqslant D_{1}^{+} \phi_{\alpha}+\rho, \\
& D_{2}^{-} Z_{\alpha}=D_{2}^{-} \phi_{\alpha}+\rho \frac{x_{1 \alpha}-x_{2 \alpha}+h}{h} \geqslant D_{2}^{-} \phi_{\alpha}, \quad D_{2}^{+} Z_{\alpha}=D_{2}^{+} \phi_{\alpha} .
\end{aligned}
$$

Recalling that $H \geqslant 0$ and using 2.1 we deduce

$$
\begin{aligned}
H_{N}\left(D_{1}^{-} Z_{\alpha}, D_{1}^{+} Z_{\alpha}, D_{2}^{-} Z_{\alpha}, D_{2}^{+} Z_{\alpha}\right) \\
=-\frac{L}{2} \sum_{k=1}^{2}\left(D_{k}^{+} Z_{\alpha}-D_{k}^{-} Z_{\alpha}\right)+H\left(\frac{D_{1}^{+} Z_{\alpha}+D_{1}^{-} Z_{\alpha}}{2}, \frac{D_{2}^{+} Z_{\alpha}+D_{2}^{-} Z_{\alpha}}{2}\right) \\
\geqslant-\frac{L}{2} \sum_{k=1}^{2}\left(D_{k}^{+} \phi_{\alpha}-D_{k}^{-} \phi_{\alpha}\right)+L \rho \geqslant-2 L L_{\phi}+L \rho \geqslant M \geqslant f\left(x_{\alpha}\right)
\end{aligned}
$$

provided that $\rho$ is sufficiently large.

Case 2: $b_{2}-x_{2 \alpha}-h \leqslant x_{1 \alpha} \leqslant b_{2}-x_{2 \alpha}$. In this case we have $x_{\alpha+e_{1}}, x_{\alpha+e_{2}} \in \Omega_{4}$ and therefore $d\left(x_{\alpha-e_{1}}\right)=x_{1 \alpha}-h, d\left(x_{\alpha+e_{1}}\right)=b_{2}-x_{2 \alpha}, d\left(x_{\alpha-e_{2}}\right)=x_{1 \alpha}, d\left(x_{\alpha+e_{2}}\right)=b_{2}-x_{2 \alpha}-h$. Thus

$$
\begin{aligned}
& D_{1}^{-} Z_{\alpha}=D_{1}^{-} \phi_{\alpha}+\rho, \quad D_{1}^{+} Z_{\alpha}=D_{1}^{+} \phi_{\alpha}+\rho \frac{b_{2}-x_{2 \alpha}-x_{1 \alpha}}{h} \leqslant D_{1}^{+} \phi_{\alpha}+\rho, \\
& D_{2}^{-} Z_{\alpha}=D_{2}^{-} \phi_{\alpha}, \quad D_{2}^{+} Z_{\alpha}=D_{2}^{+} \phi_{\alpha}+\rho \frac{b_{2}-x_{2 \alpha}-h-x_{1 \alpha}}{h} \leqslant D_{2}^{+} \phi_{\alpha} .
\end{aligned}
$$

The inequality (4.2) then follows in the same way as in Case 1.

Case 3: $x_{1 \alpha}<x_{2 \alpha}-h / 2$ and $x_{1 \alpha}<b_{2}-x_{2 \alpha}-h$. We now have $x_{\alpha \pm e_{1}}, x_{\alpha \pm e_{2}} \in \Omega_{1}$, which implies

$$
D_{1}^{ \pm} Z_{\alpha}=D_{1}^{ \pm} \phi_{\alpha}+\rho, \quad D_{2}^{ \pm} Z_{\alpha}=D_{2}^{ \pm} \phi_{\alpha}
$$

so that

$$
\begin{aligned}
H_{N}\left(D_{1}^{-} Z_{\alpha},\right. & \left.D_{1}^{+} Z_{\alpha}, D_{2}^{-} Z_{\alpha}, D_{2}^{+} Z_{\alpha}\right) \\
& =-\frac{L}{2} \sum_{k=1}^{2}\left(D_{k}^{+} \phi_{\alpha}-D_{k}^{-} \phi_{\alpha}\right)+H\left(\frac{D_{1}^{+} \phi_{\alpha}+D_{1}^{-} \phi_{\alpha}}{2}+\rho, \frac{D_{2}^{+} \phi_{\alpha}+D_{2}^{-} \phi_{\alpha}}{2}\right) \\
& \geqslant-2 L L_{\phi}+H\left(\frac{D_{1}^{+} \phi_{\alpha}+D_{1}^{-} \phi_{\alpha}}{2}+\rho, \frac{D_{2}^{+} \phi_{\alpha}+D_{2}^{-} \phi_{\alpha}}{2}\right) \geqslant M \geqslant f\left(x_{\alpha}\right)
\end{aligned}
$$

in view of (H3) provided that $\rho$ is large enough. Other points can be treated in a similar way.

\subsection{Godunov Hamiltonian}

In [2] the following formula was derived from the solution of the Riemann problem:

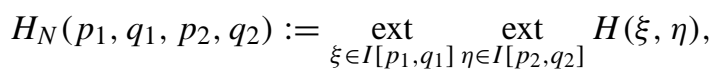


where

$$
\underset{\xi \in I[p, q]}{\operatorname{ext}}= \begin{cases}\min _{\xi \in[p, q]} & \text { if } p \leqslant q, \\ \max _{\xi \in[q, p]} & \text { if } p>q .\end{cases}
$$

Let us again verify (HN1)-(HN3).

(HN1) Clearly, $H_{N}\left(p_{1}, p_{1}, p_{2}, p_{2}\right)=H\left(p_{1}, p_{2}\right)$.

(HN2) In order to verify (3.6) and (3.7) it is sufficient to check that

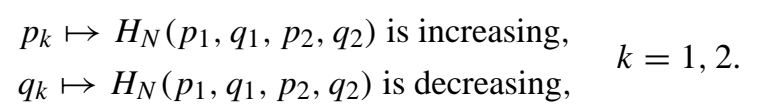

As an example, assume that $p_{1}>q_{1}$ and consider $q_{2} \mapsto H_{N}\left(p_{1}, q_{1}, p_{2}, q_{2}\right)$.

Case 1: $q_{2}<\tilde{q}_{2}<p_{2}$ :

$H_{N}\left(p_{1}, q_{1}, p_{2}, q_{2}\right)=\max _{\xi \in\left[q_{1}, p_{1}\right]} \max _{\eta \in\left[q_{2}, p_{2}\right]} H(\xi, \eta) \geqslant \max _{\xi \in\left[q_{1}, p_{1}\right]} \max _{\eta \in\left[\tilde{q}_{2}, p_{2}\right]} H(\xi, \eta)=H_{N}\left(p_{1}, q_{1}, p_{2}, \tilde{q}_{2}\right)$.

Case 2: $q_{2}<p_{2} \leqslant \tilde{q}_{2}$ :

$$
\begin{aligned}
H_{N}\left(p_{1}, q_{1}, p_{2}, q_{2}\right) & =\max _{\xi \in\left[q_{1}, p_{1}\right]} \max _{\eta \in\left[q_{2}, p_{2}\right]} H(\xi, \eta) \geqslant \max _{\xi \in\left[q_{1}, p_{1}\right]} H\left(\xi, p_{2}\right) \\
& \geqslant \max _{\xi \in\left[q_{1}, p_{1}\right]} \min _{\eta \in\left[p_{2}, \tilde{q}_{2}\right]} H(\xi, \eta)=H_{N}\left(p_{1}, q_{1}, p_{2}, \tilde{q}_{2}\right) .
\end{aligned}
$$

Case 3: $p_{2} \leqslant q_{2}<\tilde{q}_{2}$ :

$H_{N}\left(p_{1}, q_{1}, p_{2}, q_{2}\right)=\max _{\xi \in\left[q_{1}, p_{1}\right]} \min _{\eta \in\left[p_{2}, q_{2}\right]} H(\xi, \eta) \geqslant \max _{\xi \in\left[q_{1}, p_{1}\right]} \min _{\eta \in\left[p_{2}, \tilde{q}_{2}\right]} H(\xi, \eta)=H_{N}\left(p_{1}, q_{1}, p_{2}, \tilde{q}_{2}\right)$.

Other situations are treated in a similar way.

(HN3) We use the same function $Z$ as in the case of viscous regularization to verify 3.8 -3.10 . Again we examine the situation at a point $x_{\alpha}=\left(x_{1 \alpha}, x_{2 \alpha}\right) \in \Omega_{1}$.

Case 1: $x_{2 \alpha}-h \leqslant x_{1 \alpha} \leqslant x_{2 \alpha}$. Combining 4.1 with 4.6 yields

$$
H_{N}\left(D_{1}^{-} Z_{\alpha}, D_{1}^{+} Z_{\alpha}, D_{2}^{-} Z_{\alpha}, D_{2}^{+} Z_{\alpha}\right) \geqslant H_{N}\left(D_{1}^{-} \phi_{\alpha}+\rho, D_{1}^{+} \phi_{\alpha}+\rho, D_{2}^{-} \phi_{\alpha}, D_{2}^{+} \phi_{\alpha}\right) .
$$

Case 2: $b_{2}-x_{2 \alpha}-h \leqslant x_{1 \alpha} \leqslant b_{2}-x_{2 \alpha}$. Arguing as in Case 1, but using (4.3) instead of (4.1) we again derive (4.7).

Case 3: $x_{1 \alpha}<x_{2 \alpha}-h / 2$ and $x_{1 \alpha}<b_{2}-x_{2 \alpha}-h$. We infer from (4.4) that

$$
H_{N}\left(D_{1}^{-} Z_{\alpha}, D_{1}^{+} Z_{\alpha}, D_{2}^{-} Z_{\alpha}, D_{2}^{+} Z_{\alpha}\right)=H_{N}\left(D_{1}^{-} \phi_{\alpha}+\rho, D_{1}^{+} \phi_{\alpha}+\rho, D_{2}^{-} \phi_{\alpha}, D_{2}^{+} \phi_{\alpha}\right) .
$$

Thus it remains to estimate the last expression from below. Choosing a priori $\rho \geqslant 2 L_{\phi}$ we have $D_{1}^{-} \phi_{\alpha}+\rho \geqslant \rho / 2, D_{1}^{+} \phi_{\alpha}+\rho \geqslant \rho / 2$ so that with the help of (H3) we obtain

$$
H_{N}\left(D_{1}^{-} \phi_{\alpha}+\rho, D_{1}^{+} \phi_{\alpha}+\rho, D_{2}^{-} \phi_{\alpha}, D_{2}^{+} \phi_{\alpha}\right) \geqslant M \geqslant f\left(x_{\alpha}\right),
$$

provided that $\rho$ is large enough. Other points can be treated analogously. 
As a special case one obtains for the eikonal equation $H(p)=|p|$ the scheme

$$
H_{N}\left(p_{1}, q_{1}, p_{2}, q_{2}\right)=\sqrt{\left(\max \left(p_{1}^{+},-q_{1}^{-}\right)\right)^{2}+\left(\max \left(p_{2}^{+},-q_{2}^{-}\right)\right)^{2}},
$$

where $p^{+}=\max (p, 0)$ and $p^{-}=\min (p, 0)$. This scheme was examined in [13] in the context of shape-from-shading and convergence of approximations was proved with the help of a result of Barles \& Souganidis [3]. In a recent paper, Zhao [18] shows $O(|h \ln (h)|)$ convergence for this scheme when $f \equiv 1$ in the case of approximating the distance function to a point.

\section{Numerical results}

In this section we present some results of numerical calculations for 1.1), 1.2 with $H(p)=|p|$. As a first test example, let $\Omega:=(-1,1) \times(0,2)$ and $f: \Omega \rightarrow \mathbb{R}$ be defined by $f\left(x_{1}, x_{2}\right):=1$, $x_{1}<0, f\left(0, x_{2}\right):=3 / 4, f\left(x_{1}, x_{2}\right):=1 / 2, x_{1}>0$. It is not difficult to see that $f$ satisfies $(2.3)$ and one verifies that

$$
u\left(x_{1}, x_{2}\right):= \begin{cases}\frac{1}{2} x_{2}, & x_{1} \geqslant 0, \\ -\frac{\sqrt{3}}{2} x_{1}+\frac{1}{2} x_{2}, & -\frac{1}{\sqrt{3}} x_{2} \leqslant x_{1} \leqslant 0, \\ x_{2}, & x_{1}<-\frac{1}{\sqrt{3}} x_{2},\end{cases}
$$

is a viscosity solution of $|\nabla u|=f$ in the sense of Definition 2.1. Furthermore, let $\phi:=u_{\mid \partial \Omega}$. Since $H$ is globally Lipschitz continuous with constant 1 , the numerical scheme induced by viscous regularization reads: find $U: \bar{\Omega}_{h} \rightarrow \mathbb{R}$ such that

$$
\begin{aligned}
-\frac{1}{2}\left(U_{\alpha+e_{1}}+\right. & \left.U_{\alpha-e_{1}}+U_{\alpha+e_{2}}+U_{\alpha-e_{2}}-4 U_{\alpha}\right) \\
+\frac{1}{2} \sqrt{\left(U_{\alpha+e_{1}}-U_{\alpha-e_{1}}\right)^{2}+\left(U_{\alpha+e_{2}}-U_{\alpha-e_{2}}\right)^{2}} & =h f\left(x_{\alpha}\right), \quad x_{\alpha} \in \Omega_{h}, \\
U_{\alpha} & =\phi\left(x_{\alpha}\right), \quad x_{\alpha} \in \partial \Omega_{h} .
\end{aligned}
$$

The system of equations was solved with the help of Newton's method and we calculated

$$
E_{V R, h}:=\max _{x_{\alpha} \in \bar{\Omega}_{h}}\left|u\left(x_{\alpha}\right)-U_{\alpha}\right|
$$

together with the experimental order of convergence

$$
\text { eoc }=\frac{\ln \left(E_{h_{2}} / E_{h_{1}}\right)}{\ln \left(h_{2} / h_{1}\right)}
$$

for various choices of $h$. We then used the numerical Hamiltonian 4.8 to approximate the viscosity solution. Observing that

$$
\left.\max \left(\left(D_{k}^{-} U_{\alpha}\right)^{+},-D_{k}^{+} U_{\alpha}\right)^{-}\right)=\frac{1}{h}\left(U_{\alpha}-\min \left(U_{\alpha-e_{k}}, U_{\alpha+e_{k}}\right)\right)^{+}
$$

for $k=1, \ldots, n$, the discrete problem reads: find $U: \bar{\Omega}_{h} \rightarrow \mathbb{R}$ such that

$$
\begin{aligned}
\left(\sum_{k=1}^{2}\left(\left(U_{\alpha}-\min \left(U_{\alpha-e_{k}}, U_{\alpha+e_{k}}\right)\right)^{+}\right)^{2}\right)^{1 / 2} & =h f\left(x_{\alpha}\right), \quad x_{\alpha} \in \Omega_{h}, \\
U_{\alpha} & =\phi\left(x_{\alpha}\right), \quad x_{\alpha} \in \partial \Omega_{h} .
\end{aligned}
$$


The discrete solution was calculated with the help of the Fast Sweeping Method (see e.g. [18] for a description) and the corresponding errors $E_{F S, h}$ and eoc's are shown in Table 1 together with the results from the method of viscous regularization. Figure 1 shows various level lines of the solution.

TABLE 1

Absolute error in maximum norm and experimental order of convergence for the first test problem

\begin{tabular}{|c|c|c|c|c|}
\hline$h$ & $E_{V R, h}$ & eoc & $E_{F S, h}$ & eoc \\
\hline 0.1 & $1.24348 \mathrm{e}-1$ & - & $5.59016 \mathrm{e}-2$ & - \\
0.05 & $7.22984 \mathrm{e}-2$ & 0.78 & $2.79508 \mathrm{e}-2$ & 1.00 \\
0.025 & $4.08509 \mathrm{e}-2$ & 0.82 & $1.39754 \mathrm{e}-2$ & 1.00 \\
0.0125 & $2.26691 \mathrm{e}-2$ & 0.85 & $6.98771 \mathrm{e}-3$ & 1.00 \\
0.0063 & $1.24385 \mathrm{e}-2$ & 0.87 & $3.49386 \mathrm{e}-3$ & 1.00 \\
\hline
\end{tabular}

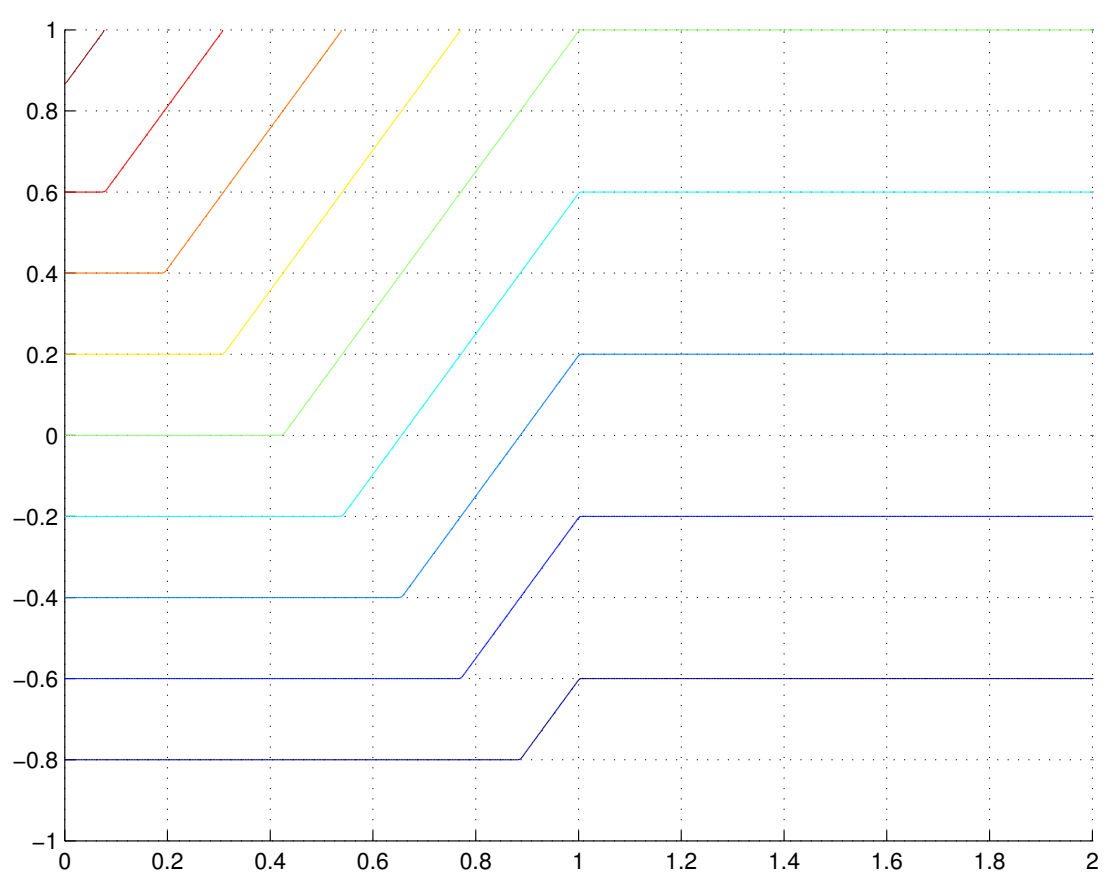

FIG. 1. Level lines of the solution from the first test problem.

We observe linear convergence in $h$ for the method [5.1), 5.2) (cf. also [18], where a one-sided bound of the form $u_{h}-u \leqslant C h \log (1 / h)$ is proved for $\left.f \equiv 1\right)$.

In our second example we consider $\Omega=(-1,1)^{2}, \phi \equiv 0$ and

$$
f\left(x_{1}, x_{2}\right):= \begin{cases}2, & \left(x_{1}-1 / 2\right)^{2}+x_{2}^{2} \leqslant 1 / 8 \text { and } x_{2} \geqslant x_{1}-1 / 2, \\ 3, & \left(x_{1}-1 / 2\right)^{2}+x_{2}^{2} \leqslant 1 / 8 \text { and } x_{2}<x_{1}-1 / 2, \\ 1, & \text { otherwise. }\end{cases}
$$


Note that in this case discontinuities of $f$ occur both along curved lines and along a straight line which is not aligned with the grid. Furthermore, the three regions, in which $f$ takes different values, meet at the triple points $(3 / 4,1 / 4),(1 / 4,-1 / 4)$. It is not difficult to check that $f$ satisfies $(2.3)$. The numerical solutions were again calculated with the help of viscous regularization and (5.1), (5.2). In the absence of an exact solution we compared the discrete solutions for various grid sizes with an approximation $U_{f}$ on a fine grid $(h=1 / 640)$. The results are displayed in Table 2, while Figure 2 shows some level curves of the solution.

TABLE 2

Absolute error in maximum norm and experimental order of convergence for the second test problem

\begin{tabular}{|c|c|c|c|c|}
\hline$h$ & $E_{V R, h}$ & eoc & $E_{F S, h}$ & eoc \\
\hline 0.1 & $9.07922 \mathrm{e}-2$ & - & $1.10429 \mathrm{e}-1$ & - \\
0.05 & $1.29179 \mathrm{e}-1$ & -0.51 & $1.28365 \mathrm{e}-1$ & -0.22 \\
0.025 & $1.04327 \mathrm{e}-1$ & 0.31 & $8.68148 \mathrm{e}-2$ & 0.56 \\
0.0125 & $7.35184 \mathrm{e}-2$ & 0.50 & $5.00901 \mathrm{e}-2$ & 0.79 \\
0.0063 & $4.33351 \mathrm{e}-2$ & 0.76 & $2.65873 \mathrm{e}-2$ & 0.91 \\
\hline
\end{tabular}

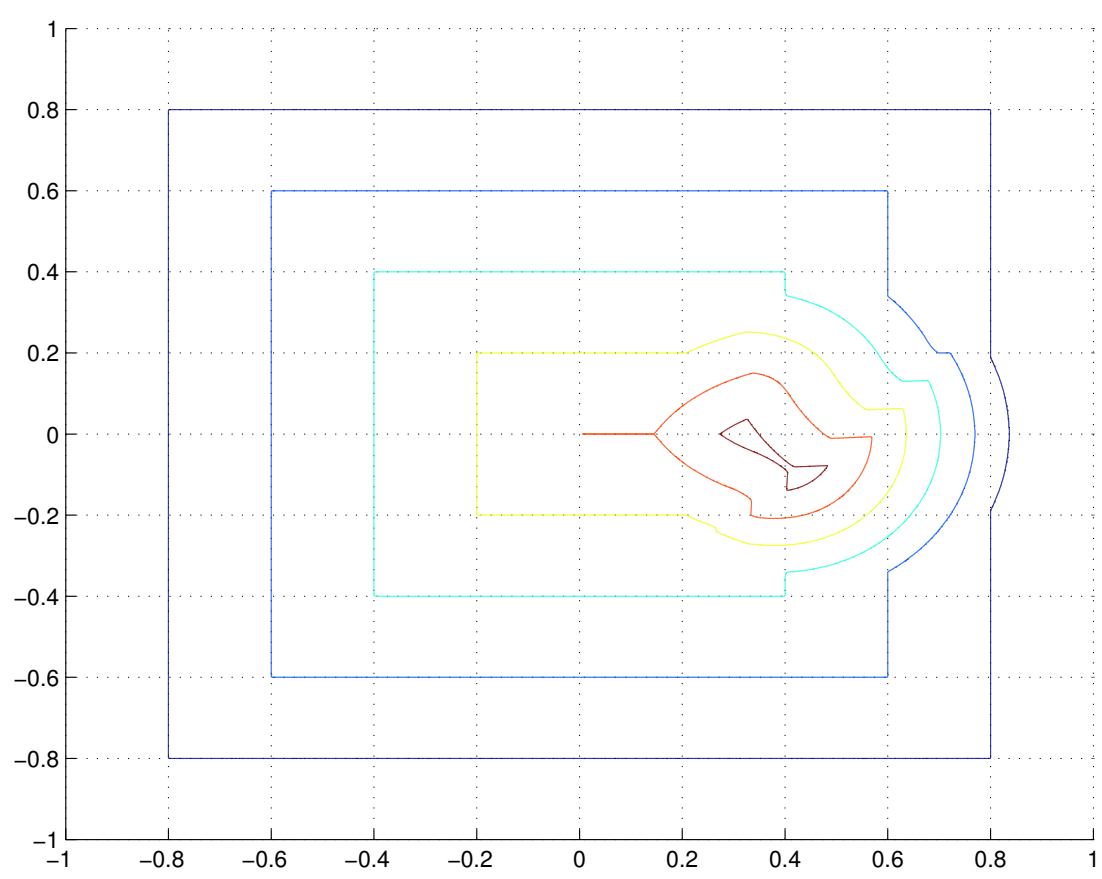

FIG. 2. Level lines of the solution from the second test problem.

Acknowledgments. Part of this work was carried out while the authors participated in the 2003 Programme Computational Challenges in Partial Differential Equations at the Isaac Newton Institute, Cambridge, UK. 


\section{REFERENCES}

1. Bardi, M. \& CApuzzo-Dolcetta, I. Optimal Control and Viscosity Solutions of Hamilton-JacobiBellman Equations. Birkhäuser Boston (1997). Zbl 0890.49011 MR 1484411

2. BARDI, M. \& OSHER, S. The nonconvex multi-dimensional Riemann problem for Hamilton-Jacobi equations. SIAM J. Math. Anal. 22 (1991), 344-351. Zbl 0733.35073 MR 1084960

3. BARles, G. \& Souganidis, P. E. Convergence of approximation schemes for fully nonlinear second order equations, Asymptotic Anal. 4 (1991), 271-283. Zbl 0729.65077 MR 1115933

4. CAmilli, F. \& Siconolfi, A. Hamilton-Jacobi equations with measurable dependence on the state variable. Adv. Differential Equations 8 (2003), 733-768. Zbl 1036.35052

5. Crandall, M. G. \& Lions, P. L. Viscosity solutions of Hamilton-Jacobi equations. Trans. Amer. Math. Soc. 277 (1983), 1-42. Zbl 0599.35024 MR 0690039

6. Crandall, M. G. \& Lions, P. L. Two approximations of solutions of Hamilton-Jacobi equations. Math. Comp. 43 (1984), 1-19. Zbl 0556.65076

7. Falcone, M. \& Ferretti, R. Semi-Lagrangian schemes for Hamilton-Jacobi equations, discrete representation formulae and Godunov methods. J. Comput. Phys. 175 (2002), 559-575. Zbl 1007.65060 MR 1880118

8. IsHII, H. Hamilton-Jacobi equations with discontinuous Hamiltonians on arbitrary open sets. Bull. Fac. Sci. Engrg. Chuo. Univ. 28 (1985), 33-77. Zbl 0937.35505 MR 0845397

9. IsHit, H. A simple, direct proof of uniqueness for solutions of the Hamilton-Jacobi equations of eikonal type, Proc. Amer. Math. Soc. 100 (1987), 247-251. Zbl 0644.35017 MR 0884461

10. NewComb II, R. T. \& Su, J. Eikonal equations with discontinuities. Differential Integral Equations 8 (1995), 1947-1960. Zbl 0854.35022 MR 1348959

11. Osher, S. \& FedKIW, R. Level Set Methods and Dynamic Implicit Surfaces. Appl. Math. Sci. 153, Springer, New York (2003). Zbl 1026.76001 MR 1939127

12. Ostrov, D. Viscosity solutions and convergence of monotone schemes for synthetic aperture radar shape-from-shading equations with discontinuous intensities. SIAM J. Appl. Math. 59 (1999), 2060-2085. Zbl 0936.35048 MR 1709797

13. RouY, E. \& TOURIN, A. A viscosity solutions approach to shape-from-shading. SIAM J. Numer. Anal. 29 (1992), 867-884. Zbl 0754.65069 MR 1163361

14. Sethian, J. A. Fast marching methods. SIAM Rev. 41 (1999), 199-235. Zbl 0926.65106 MR 1684542

15. SORAVIA, P. Boundary value problems for Hamilton-Jacobi equations with discontinuous Lagrangian. Indiana Univ. Math. J. 51 (2002), 451-477. Zbl 1032.35055 MR 1909297

16. Tourin, A. A comparison theorem for a piecewise Lipschitz continuous Hamiltonian and applications to shape-from-shading. Numer. Math. 62 (1992), 75-85. Zbl 0762.65087 MR 1159046

17. Tsai, Y.-H., Cheng, L.-T., Osher, S. \& ZhaO, H. Fast sweeping algorithms for a class of HamiltonJacobi equations. SIAM J. Numer. Anal. 41 (2003), 673-694. Zbl pre02027739 MR 2004194

18. ZhAO, H. Fast sweeping method for eikonal equations. Math. Comp. (to appear). 